\title{
High Frequency Guided Waves for Disbond Detection in Multi-Layered Structures
}

\author{
Paul Fromme ${ }^{1)}$, Jean-Pascal Reymondin ${ }^{2)}$, Bernard Masserey ${ }^{3)}$ \\ 1) Department of Mechanical Engineering, University College London, London WC1E 7JE, United Kingdom. \\ p.fromme@ucl.ac.uk \\ 2) Department of Systems Engineering, School of Business and Engineering Vaud, Yverdon, Switzerland \\ 3) Department of Mechanical Engineering, University of Applied Sciences and Arts, Fribourg, Switzerland
}

\begin{abstract}
Summary
Aerospace structures often contain multi-layered metallic components where hidden defects such as localized disbonds can develop, necessitating non-destructive testing. Model structures consisting of two adhesively bonded aluminium plates and artificial defects in the bond layer were manufactured. Immersion ultrasonic C- scans were used to check the uniformity of the bond layer and the manufactured defects. Employing standard wedge transducers, high frequency guided ultrasonic wayes that penetrate through the complete specimen thickness were generated. Interference occurs between the wave modes during propagation along the structure, resulting in a frequency dependent variation of the energysthrough the thickness with distance. Significant propagation distance with a strong, non-dispersive main wave pulse was achieved. The interaction of the high frequency guided ultrasonic waves with small disbonds in the sealant layer and lack of sealant in the multilayer structure was investigated. Guided wave pulse-echo measurements were conducted to verify the detection sensitivity and the influence of the stand-off distance predictedfrom the finite element simulations. The results demonstrated the potential of high frequency guided waves for hidden defect detection at critical and difficult to access locations in aerospace structures from a stand-off distance.
\end{abstract}

PACS no. 43.20.Mv, 43.35.Zc, 43.20.Gp

\section{Introduction}

Damage such as fatigue cracks and disbonds in the sealant layers connecting multiple metallic layers can occur during the service life of aircraft structures due to environmental and cyclic loading conditions [1]. Defect detection in all layers of multi-layered aircraft structures is a requirement for future Structural Health Monitoring (SHM) systems [2]. Ultrasonic testing (UT) techniques using an angled phase-array probe and automated analysis of the acquired ultrasonic signals for 2nd layer defect detection have been developed [3]. Pulse-echo and transmission bulk ultrasonic techniques have been used to detect defects in bonded joints [4]. However, defect detection in the 2 nd layer using conventional, bulk UT remains one of the challenges for aircraft maintenance and inspection, especially if the sealant (coupling medium) between the layers is inadequate or missing [3].

Guided ultrasonic waves allow for the inspection and monitoring of large structures as they can propagate long distances in plate structures [5]. Often the excitation frequency is chosen in the low range below the cut-off fre-

Received 2 May 2017,

accepted 29 September 2017. quency of the higher wave modes to simplify data interpretation [6]. This results in wavelengths that are significantly larger than in bulk wave UT, thus limiting the sensitivity for the detection of small defects [7]. The propagation of low frequency guided ultrasonic waves in bonded components has been studied [8]. Feature-guided waves were used to monitor adhesive curing for bonded stiffeners [9]. Artificial disbonds and incomplete curing of adhesively bonded joints were monitored using the fundamental Lamb wave modes $[10,11]$. Fatigue crack growth at a fastener hole in a multi-layered structure was monitored using low frequency guided ultrasonic waves [12]. The potential for defect detection in an inaccessible layer was demonstrated, but limited sensitivity was found for small cracks.

Non-destructive testing using guided ultrasonic wave modes in the higher frequency-thickness range has been investigated. The $\mathrm{S}_{0}$ mode (around $5 \mathrm{MHz} \mathrm{mm}$ ) was employed for corrosion detection in aircraft structures [13], and longitudinal modes (above $15 \mathrm{MHz} \mathrm{mm}$ ) were used for plate inspection [14]. In the frequency range up to $20 \mathrm{MHz}$ mm the $\mathrm{A}_{1}$ Lamb wave mode was excited using a contact wedge transducer [15] and higher order mode clusters of guided waves were excited using EMATs [16]. Standard pulse-echo $(\mathrm{P} / \mathrm{E})$ measurements using a combination of the $A_{0}$ and $S_{0}$ modes at $6.75 \mathrm{MHz}$ mm allowed 
for the determination of the location of small surface defects [17]. Structures can be inspected over reasonably long distances, and these guided wave modes be used even if local access to the inspected part is not possible [6]. The wavelengths of the higher frequency guided wave modes are typically in the low millimeter range, allowing good sensitivity for the detection of small defects [14]. Guided ultrasonic wave modes have energy distributed through the thickness of the multi-layered structure, allowing for the inspection of the different layers and sealant. Fatigue crack detection in multi-layered structures using high frequency guided ultrasonic waves $(5 \mathrm{MHz})$ has been investigated [18]. The potential for defect detection was demonstrated, with detection sensitivity depending on the interface conditions between the layers. Attenuation of the high frequency guided ultrasonic waves due to the viscoelastic material (e.g. adhesive) between the metallic layers was noted, making the monitoring of large areas more difficult. Guided interface waves were employed for the evaluation of adhesive joints [19] and for adhesively bonded multilayer structures [20]. Fatigue cracks in the bottom layer of multi-layered tensile specimens were detected using P/E measurements from a stand-off distance [21].

In this contribution, high frequency guided ultrasonic waves were employed for the detection of manufactured defects in the sealant layer of multi-layered aerospace structures, consisting of two adhesively bonded aluminium plates with an epoxy based sealant layer [22]. The quality of the sealant layer and the defect detectability were investigated from an ultrasonic immersion $\mathrm{C}$-scan. High frequency guided wave modes $\left(\mathrm{A}_{0}\right.$ and $\mathrm{S}_{0}$ modes at $6.25 \mathrm{MHz} \mathrm{mm}$ ) were excited and measured using a standard $90^{\circ}$ angle beam transducer in a P/E configuration. These modes can propagate over medium distances and are sensitive for defect detection through the complete specimen thickness. Their wave propagation characteristics in the multi-layered model structure have been studied previously [23]. 2D Finite Element (FE) simulations have been carried out and compared to laboratory measurements. The sensitivity for the detection of missing sealant and artificial disbonds from a stand-off distance has been investigated.

\section{Experiments}

The multi-layered structures were built using two $3 \mathrm{~mm}$ thick aluminium plates with a width of $70 \mathrm{~mm}$ and a length of $600 \mathrm{~mm}$, connected with an approximately $0.25 \mathrm{~mm}$ thick epoxy based sealant layer (Figure 1). The plate material was an aluminium alloy 2014 T6 widely used for aerospace applications, having a Young's modulus of $73.1 \mathrm{GPa}$, Poisson's ratio of 0.33 , and density of $2800 \mathrm{~kg} / \mathrm{m}^{3}$.

The sealant was a two-part structural paste adhesive Hysol EA 9394 with a Young's modulus of $4.237 \mathrm{GPa}$, density of $1360 \mathrm{~kg} / \mathrm{m}^{3}$, and Poisson's ratio of 0.45 (manufacturer data [24]).The thickness of the sealant layer was controlled by mixing approximately $4 \%$ volume fraction

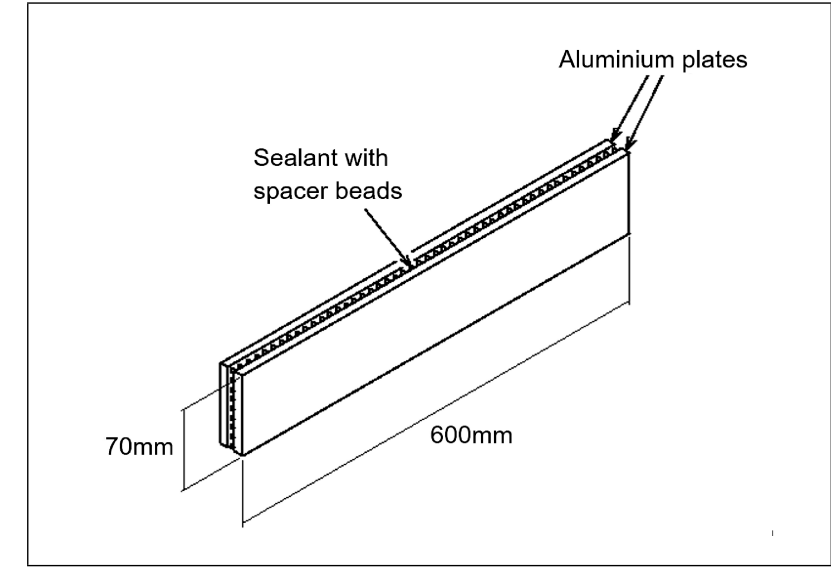

Figure 1. Geometry of the manufactured multi-layered structure.

Table I. Overview of specimens with manufactured defects.

\begin{tabular}{|lcc|}
\hline Defect type & $\begin{array}{c}\text { Defect size } \\
{[\mathrm{mm} \times \mathrm{mm}]}\end{array}$ & $\begin{array}{c}\text { Avg. sealant thickness } \\
{[\mathrm{mm}]}\end{array}$ \\
\hline Lack of sealant & $10 \times 70$ & 0.25 \\
Disbond & $10 \times 70$ & 0.25 \\
Disbond & $15 \times 15$ & 0.27 \\
Disbond & $10 \times 10$ & 0.27 \\
\hline
\end{tabular}

of spacer beads with a maximum diameter of $0.249 \mathrm{~mm}$ into the epoxy paste and clamping the specimen during curing at room temperature.

Two specimens without defects and 4 specimens with different artificial defects were manufactured and the sealant thickness measured (Table I). Several solutions to create a disbond defect in a multilayer structure were initially tested. The selected method consists of a combination of an aluminium foil and an optical film. An optical lens cleaner film is a thin film of approximately $0.01 \mathrm{~mm}$ thickness used to clean camera lenses. When coated with an aluminium foil, the optical film confines air into the fine fabric weaves and separates the aluminium foil surfaces. The aluminium foil of $0.013 \mathrm{~mm}$ thickness prevents the sealant to soak into the optical film and is expected to ensure a disbond between the bonded aluminium plates. Figure 2 shows the folding of the aluminium foil around the optical film.

In Figure 3 different sizes of disbond defects are shown. Sealant was applied on both internal faces of the aluminium plates composing the multilayer structure. The disbond defects were placed directly on the sealant at a distance of $150 \mathrm{~mm}$ from the plate end, as shown in Figure 4 . The disbond defects are located approximately in the center of the multilayer thickness.

The quality of the sealant layer and the positioning and detectability of the artificial defects were investigated using a conventional immersion scanning system. The multilayered specimens were immersed in a water tank and Cscans were performed with a step size of $0.2 \mathrm{~mm} \times 0.2 \mathrm{~mm}$ using a $15 \mathrm{MHz}$ broad band, quarter inch immersion transducer with a focal length of 1.5 inches. 


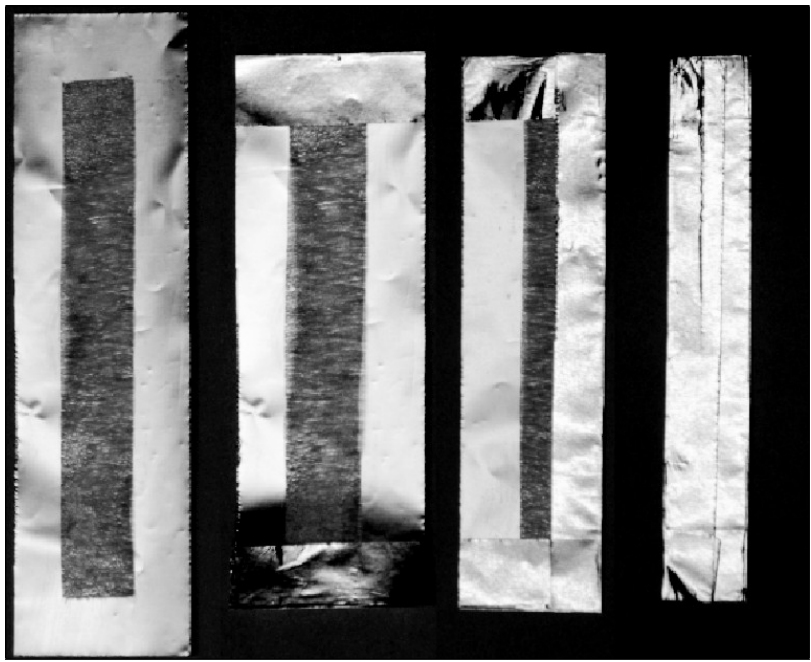

Figure 2. Folding of aluminum foil around optical lens cleaner film to create disbond.

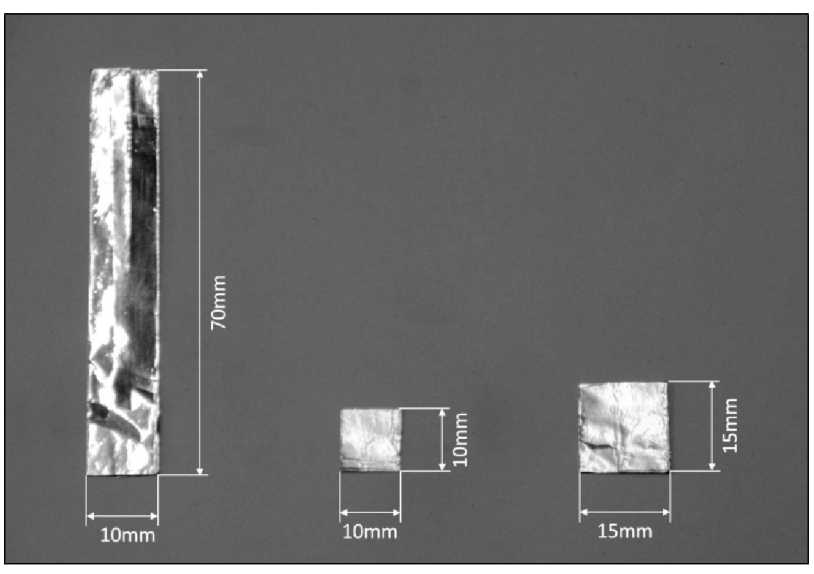

Figure 3. Different sizes of disbonds: $10 \mathrm{~mm} \times 70 \mathrm{~mm} ; 10 \mathrm{~mm} \times$ $10 \mathrm{~mm}, 15 \mathrm{~mm} \times 15 \mathrm{~mm}$.

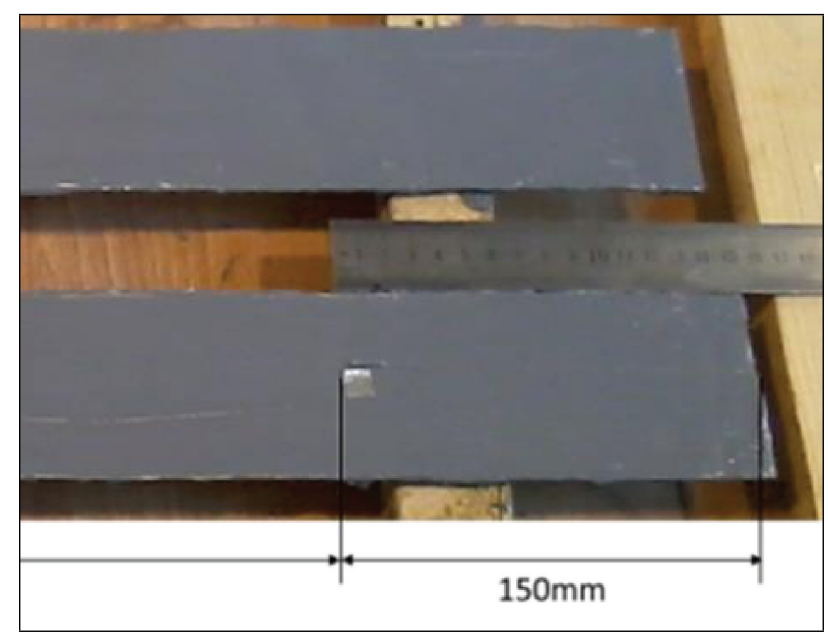

Figure 4. Disbond defect $10 \mathrm{~mm} \times 10 \mathrm{~mm}$ placed $150 \mathrm{~mm}$ from end of multi-layered specimen.

The high frequency guided ultrasonic waves were excited by placing a standard $1 \mathrm{MHz}$ half inch transducer mounted on a $90^{\circ}$ angle beam wedge for steel on one of

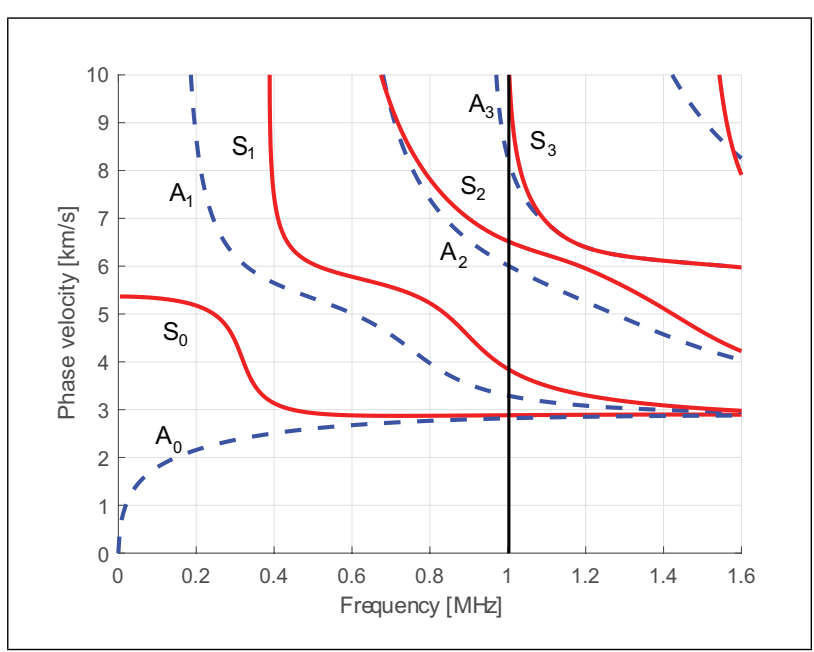

Figure 5. (Colour online) Phase velocity dispersion diagram for multi-layered structure ( $3 \mathrm{~mm}$ aluminium, $0.25 \mathrm{~mm}$ sealant, $3 \mathrm{~mm}$ aluminium); $1 \mathrm{MHz}$ excitation frequency marked.

the aluminium surfaces. Using liquid couplant the wedge was aligned so that the main propagation axis was along the specimen center line. The wedge was manually placed on the specimen surface and measurements were taken in $10 \mathrm{~mm}$ intervals for stand-off distances ranging between 50 and $270 \mathrm{~mm}$ from the defect location. The reflection at the defects was measured using a standard ultrasonic pulser-receiver, driving the transducer with a voltage spike and using the inbuilt receiver circuitry.

\section{Guided wave propagation}

The dispersion diagram of the $6.25 \mathrm{~mm}$ thick multi-layered structure was calculated using Disperse [25] and is shown in Figure 5. The guided wave modes were named in analogy to Lamb modes in a single layer plate, according to the naming convention from Royer and Dieulesaint [26]: the index corresponds to the number of nodes in the plate thickness at cut-off frequency for in-plane displacement (odd anti-symmetric modes $\mathrm{A}_{2 n+1}$ and even symmetric modes $\mathrm{S}_{2 n}$ ) and out-of-plane displacement (even antisymmetric modes $\mathrm{A}_{2 n}$ and odd symmetric modes $\mathrm{S}_{2 n+1}$ ), where $n$ is an integer greater than or equal to zero [23]. Using a $1 \mathrm{MHz}, 90^{\circ}$ angle beam transducer leads to a stress field at the specimen surface (interface with wedge) with a spatial periodicity of about $3 \mathrm{~mm}$, corresponding to a phase velocity of $3000 \mathrm{~m} / \mathrm{s}$.

At this frequency, above the cut-off frequency of the higher modes, the fundamental modes $\mathrm{A}_{0}$ and $\mathrm{S}_{0}$ have phase velocities of $2819 \mathrm{~m} / \mathrm{s}$ and $2883 \mathrm{~m} / \mathrm{s}$ and show low dispersive behavior. The higher $A_{1}$ and $S_{1}$ modes have phase velocities of $3295 \mathrm{~m} / \mathrm{s}$ and $3852 \mathrm{~m} / \mathrm{s}$, and exhibit significantly more dispersion. Based on the phase velocities of the different modes, preferential excitation of the $\mathrm{A}_{0}$, $\mathrm{S}_{0}$, and $\mathrm{A}_{1}$ mode was predicted, with larger phase velocity difference for the other modes, thus not expected to be significantly generated.

The mode shapes of the first four modes $\left(\mathrm{A}_{0}, \mathrm{~S}_{0}, \mathrm{~A}_{1}\right.$, and $S_{1}$ ) at $1 \mathrm{MHz}$ are illustrated in Figure 6 . The anti- 
symmetric mode $\mathrm{A}_{0}$ can be interpreted as the individual, in-phase bending of the aluminium layers with almost constant out-of-plane displacement and significant in-plane displacement gradient in the sealant layer (Figure $6 \mathrm{a}$ ). The $\mathrm{S}_{0}$ mode shows out-of-phase bending (opposite directions) of the aluminium layers, with the structure stretched and compressed in the wave propagation direction and the sealant layer subjected to a significant out-ofplane displacement gradient (Figure $6 \mathrm{~b}$ ). The $\mathrm{A}_{1}$ and $\mathrm{S}_{1}$ modes are associated with stretching and compression of the individual aluminium plates.

For the $\mathrm{A}_{1}$ mode, the deformations between upper and lower plates are out-of-phase (Figure $6 \mathrm{c}$ ), while they are in-phase for the $S_{1}$ mode (Figure $6 \mathrm{~d}$ ). All four modes show significant energy and energy gradient within the sealant layer, thus good sensitivity is expected for defects located within the sealant.

The wave propagation in the undamaged multi-layered specimens was measured using a laser interferometer and compared to results from a FE simulation and theoretical predictions in a previous publication [23]. Results are briefly summarized below. Measured time traces showed a clear and sharp first pulse with very limited dispersion, which was identified from the group velocities and 2D FFT as consisting of the two fundamental modes $A_{0}$ and $S_{0}$ with theoretical group velocities at $1 \mathrm{MHz}$ of $3035 \mathrm{~m} / \mathrm{s}$ and $2940 \mathrm{~m} / \mathrm{s}$ respectively. A second, slower pulse was identified as the $A_{1}$ mode with a theoretical group velocity of approximately $2170 \mathrm{~m} / \mathrm{s}$. This pulse showed significant dispersion and decreased in amplitude with propagation distance. The $S_{1}$ mode was not observed experimentally with any significant amplitude. It was found that the relative amplitudes of the wave modes depend on the excitation wedge transducer, but that the measured phase velocities exhibit a difference of less than $1 \mathrm{~m} / \mathrm{s}(0.03 \%)$.

Constructive and destructive interferences occurred due to the differences in phase velocity, and beating effects as described in [23]. The beatlength of two wave modes is inversely proportional to the difference between their wavenumbers [27]. This generated energy exchange between the two aluminium plates and thus an insonification of the complete specimen thickness. A clear guided wave pulse could be observed $300 \mathrm{~mm}$ from the transducer location, indicating the applicability for defect detection over medium range distances along multi-layered plate specimens.

\section{Finite Element Simulation}

The wave propagation and scattering at sealant defects in the multi-layer structure was simulated using ABAQUS/ Explicit, based on an explicit dynamic Finite Element (FE) formulation. A two-dimensional model representing the side view of the specimen was used, assuming plane strain wave propagation (Figure 7). The model had a structured mesh using 4 node linear, plane strain, square elements. In general, ten elements per wavelength are required as a minimum for correct wave propagation modelling [28].

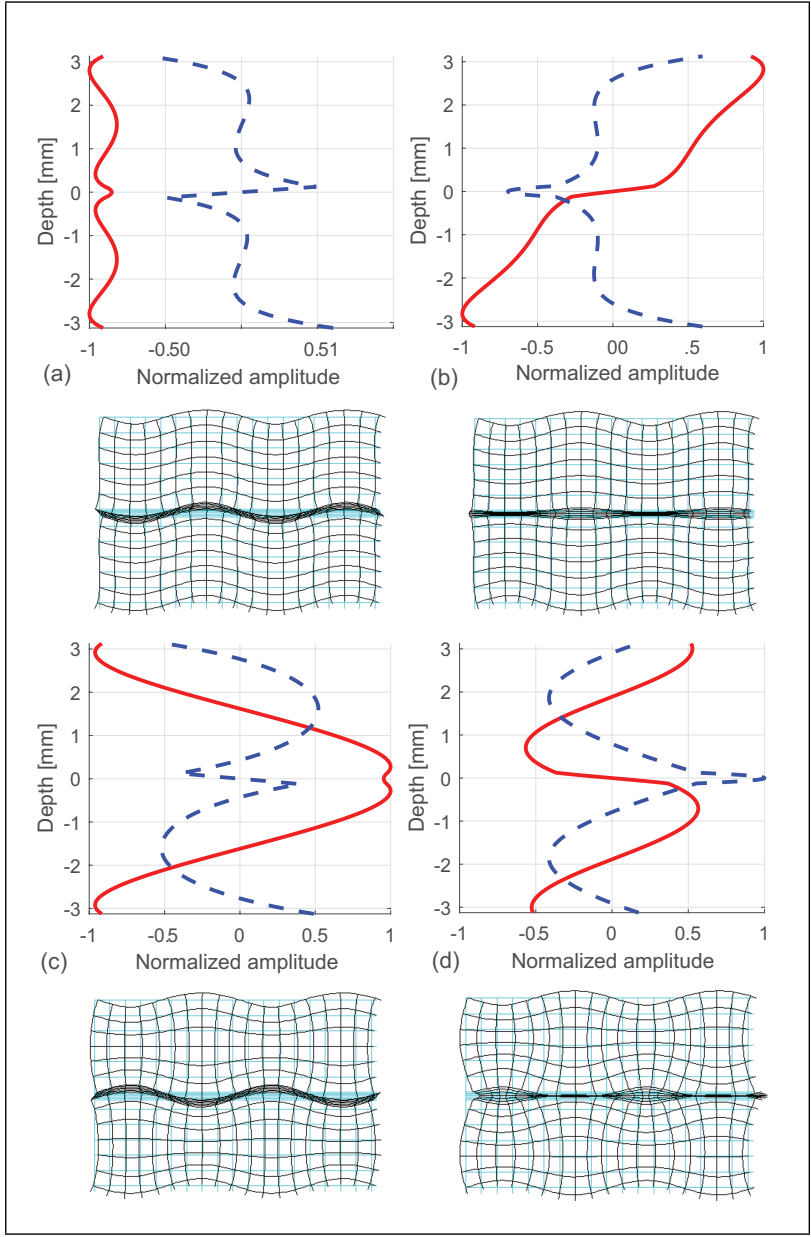

Figure 6. (Colour online) Mode shapes in multi-layered plate at $1 \mathrm{MHz}$ frequency; in-plane (dashed blue) and out-of-plane (solid red) displacement components, deformation shape: a) $\mathrm{A}_{0}$ mode, b) $S_{0}$ mode, c) $A_{1}$ mode, d) $S_{1}$ mode.

For guided wave mode propagation and scattering simulations, higher spatial sampling is generally used to ensure accurate modelling of the stress and displacement fields through the thickness of the specimen [29].

An element size of $50 \mu \mathrm{m}$ was therefore selected, corresponding to approximately $1 / 60$ of the wavelength of the fundamental Lamb modes at $1 \mathrm{MHz}$. The chosen element size results in a numerical error below $0.01 \%$ for the phase velocity of the fundamental modes $\mathrm{A}_{0}$ and $\mathrm{S}_{0}$ and an error of less than $1 \%$ for the corresponding beatlength [23]. Rayleigh damping was used in the FE analysis to account for the measured beam spread and the material damping in the sealant layer.

The two-dimensional model was modified to represent the two $10 \mathrm{~mm} \times 70 \mathrm{~mm}$ defects across the width of the sealant. In 2D, simulation of the smaller, square disbond defects is not possible. For the $10 \mathrm{~mm}$ long missing sealant defect, the sealant elements at the defect position were removed, assuming free boundary conditions on the defect contour. For the $10 \mathrm{~mm}$ long disbond, two neighbouring lines of elements along the sealant layer were modelled as elements that do not share nodes in the defect zone and thus are not connected along the disbond line. Since the 


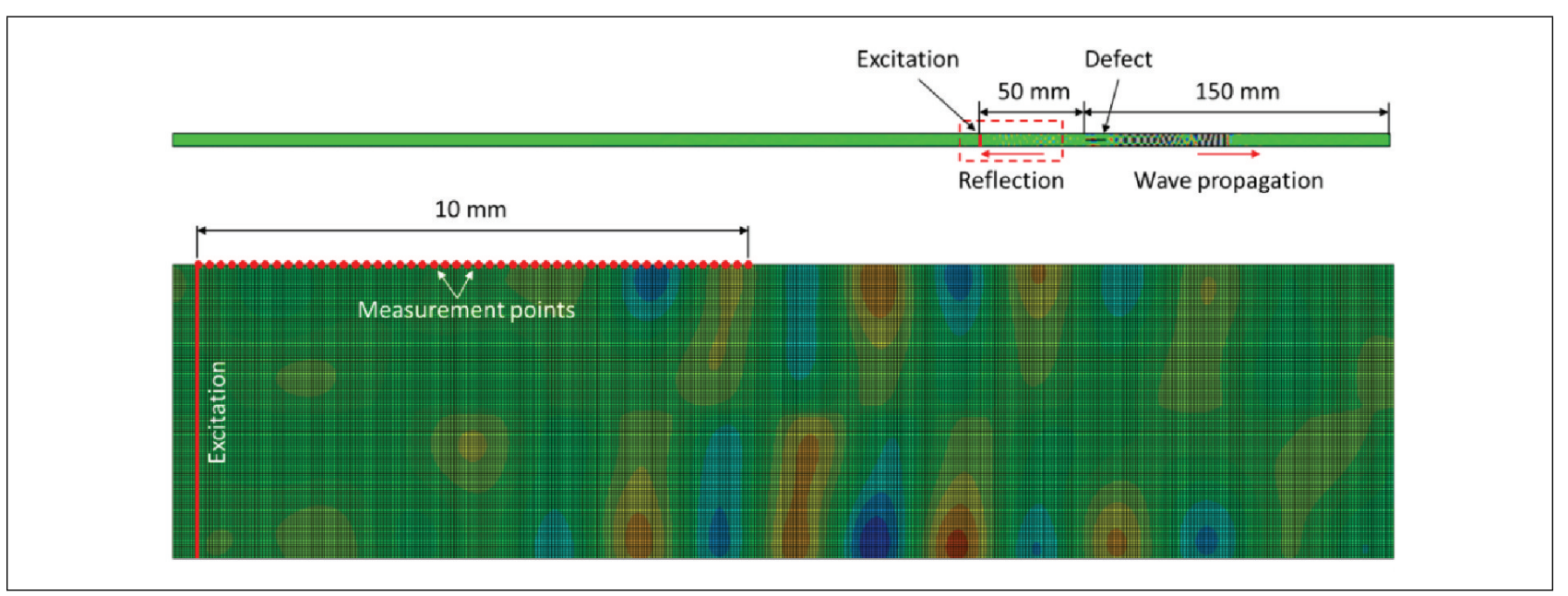

Figure 7. (Colour online) 2D FE model of multi-layered structure with defect located $150 \mathrm{~mm}$ from end; excitation and monitoring locations shown.

$0.25 \mathrm{~mm}$ sealant thickness is discretized with 5 elements, the separation is not exactly centered, with three sealant elements below and two sealant elements above the disbond line.

Excitation was obtained by specifying the in-plane and out-of-plane displacement components (shifted by a phase angle of $\pi / 2$ ) on a vertical line through the specimen thickness. The displacement components were determined by addition of the normalized mode shapes of the first 3 Lamb wave modes at the center frequency of $1 \mathrm{MHz}$, obtained from Disperse [25], after weighting with the relative mode amplitudes obtained from previous measurements [23]. The profiles were multiplied with a four cycle sinusoid (center frequency $1 \mathrm{MHz}$ ) in a Hanning window, to approximate the observed signal of the angle beam transducer driven by a voltage pulse. The time-dependent displacement components were specified as boundary conditions on a vertical line. The adjacent line on one side was fixed to achieve wave propagation in one direction only. The boundary conditions were removed after excitation to allow for reflections to pass through. The predicted outof-plane displacement component along the propagation direction was compared with the laser measurements on the center line of a multilayered specimen without defect to verify the wave propagation characteristics [23].

The out-of-plane displacement at the specimen surface was recorded over a length of $10 \mathrm{~mm}$ (50 nodes in $0.2 \mathrm{~mm}$ steps) to approximate the received P/E signal for the angle beam transducer footprint (Figure 7). The average of the signals from the multiple monitoring points was calculated after shifting the signals in the time domain to account for the phase shift between the monitoring points [29]. The maximum amplitude for each stand-off distance to the defect was extracted from the time-signal after separation using a time-window.

\section{Ultrasonic immersion C-scan}

Ultrasonic immersion C-scans were conducted for all of the specimens and evaluated on the basis of two time gates.
The maximum amplitude of the fully rectified signal in each time gate was displayed and analyzed as a function of the specimen position. The first echo observed on the A-scan for each grid point corresponds to the top of the upper aluminium plate and was used to synchronize the time base.

The second echo corresponds to the back-wall echo of the upper aluminum layer. A first time gate was set to extract the maximum amplitude from this reflection, indicating a loss of transmission into the sealant layer. The next echo arises from the reflection at the interface between the sealant and bottom aluminium layer for the ultrasonic wave having propagated through the sealant. On a properly bonded area, it is possible to observe a succession of echoes, a sign of energy transmission into the sealant. The second time gate allows for the evaluation of the maximum amplitude of this echo.

The ultrasonic immersion C-scans for the $10 \mathrm{~mm} \times$ $70 \mathrm{~mm}$ lack of sealant and disbond are shown in Figure 8. The lack of sealant is clearly detected from the C-scan measurement. Within the defect zone the amplitude of the reflection at the upper sealant interface increases, indicating a higher impedance mismatch with air (Figure 8a). Small areas $\left(<2 \mathrm{~mm}^{2}\right)$ of higher amplitude can be seen outside of the defect zone, due to inclusions in the sealant arising from the manual manufacturing process. The $\mathrm{C}$ scan based on the second time gate shows a uniform region of low amplitude (Figure 8c), as the ultrasonic wave cannot propagate across the missing sealant. The variation of the amplitude in the non-damaged areas gives an indication that some variation in the sealant layer exists (thickness or material properties), but that overall the sealant layer is reasonably homogeneous.

For the artificial disbond, variations of amplitudes in the ultrasonic C-scans are more difficult to observe. The $\mathrm{C}$-scan results for the disbond across the specimen width are displayed in Figure 8b and 8d. There is no clear backwall echo from the upper aluminium plate, as a significant part of the ultrasonic energy is transmitted into the 


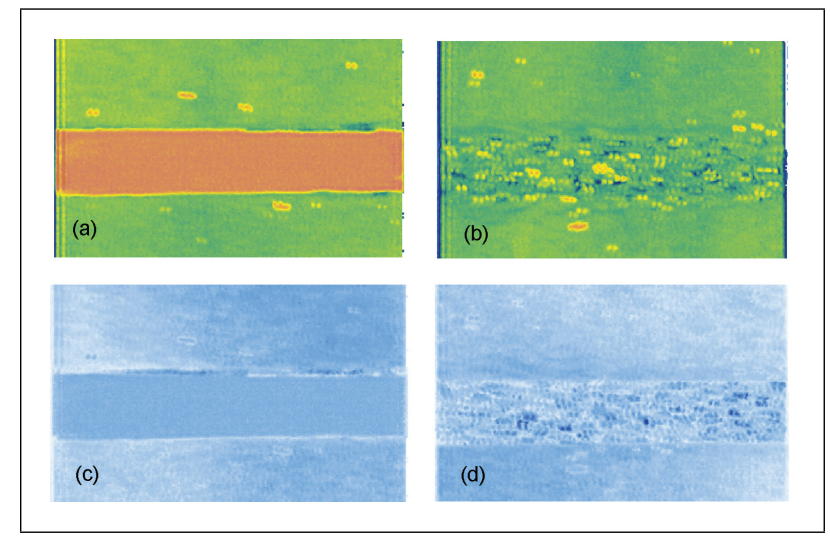

Figure 8. (Colour online) Ultrasonic immersion C-scan, $15 \mathrm{MHz}$ frequency, step size $0.2 \mathrm{~mm}$; maximum amplitude (colour-coded) of time gate, $10 \mathrm{~mm} \times 70 \mathrm{~mm}$ defects; reflection at upper sealant interface for a) lack of sealant, b) disbond, and reflection at bottom aluminium layer for c) lack of sealant and d) disbond.

sealant layer. The defect zone is characterized by small areas with higher and lower amplitudes, showing that part of the energy propagates through the disbond. Nevertheless, the defect contour is recognizable from the variation of amplitude across the disbond area, characterized by multiple maxima and minima.

The ultrasonic C-scans are shown in Figure 9 for the square artificial disbonds. As for the larger disbond, the defect zone for the first time gate is characterized by an area with multiple maxima and minima across the disbond area (Figure $9 \mathrm{a} / \mathrm{b}$ ). The defect contour is recognizable, but shows up more clearly for the second time gate, as lower reflection amplitude from the bottom of the sealant layer (Figure 9c/d). Figure 9a/b show areas of higher amplitude, probably due to an inclusion or void in the sealant. All manufactured defects could be detected from the ultrasonic immersion C-scans. Some variations of the sealant layer were observed, but only very limited larger or discrete imperfections. This standard non-destructive method allowed good resolution of the damage shape and location in line with literature [4], but is rather time-consuming and required immersion of the specimens in the test rig.

\section{Pulse-echo measurement of damage}

As described in Section 2, pulse-echo measurements were conducted using the $1 \mathrm{MHz}$ wedge transducer to excite the guided wave modes at different stand-off distances from the defects. Figure 10 shows a typical time trace, in this case for the measurement of the $10 \mathrm{~mm} \times 70 \mathrm{~mm}$ disbond. Initial reverberations within the wedge (until $0.05 \mathrm{~ms}$ ) limit the minimum stand-off distance for defect detection. The pulse reflected at the end of the specimen at $0.22 \mathrm{~ms}$ is not shown to full scale.

Strong end reflections were observed for all measurements, indicating that a significant part of the incident wave pulse was transmitted past the defect and good wave propagation achieved. The reflected pulse at the disbond can be clearly observed at $0.13 \mathrm{~ms}$ (between vertical lines)
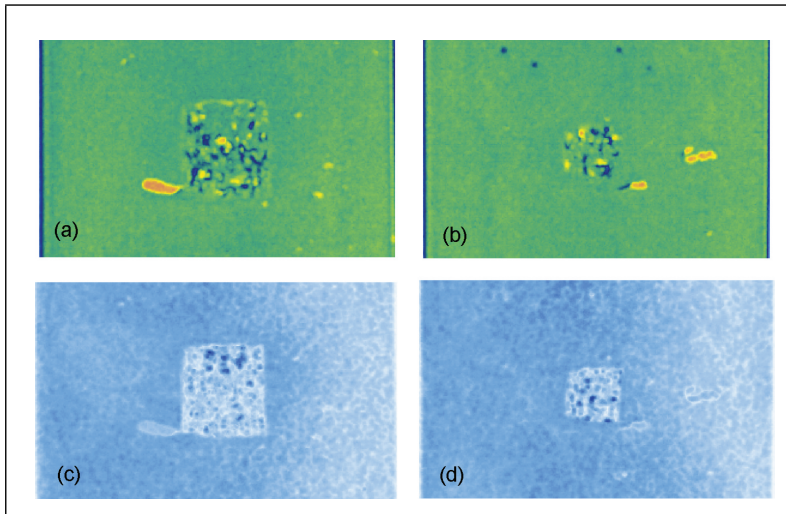

Figure 9. (Colour online) Ultrasonic immersion C-scan, $15 \mathrm{MHz}$ frequency, step size $0.2 \mathrm{~mm}$; maximum amplitude (colour-coded) of time gate; reflection at upper sealant interface for a) $15 \mathrm{~mm}$ and b) $10 \mathrm{~mm}$ squared disbonds, and reflection at bottom aluminium layer for c) $15 \mathrm{~mm}$ and d) $10 \mathrm{~mm}$ squared disbonds.

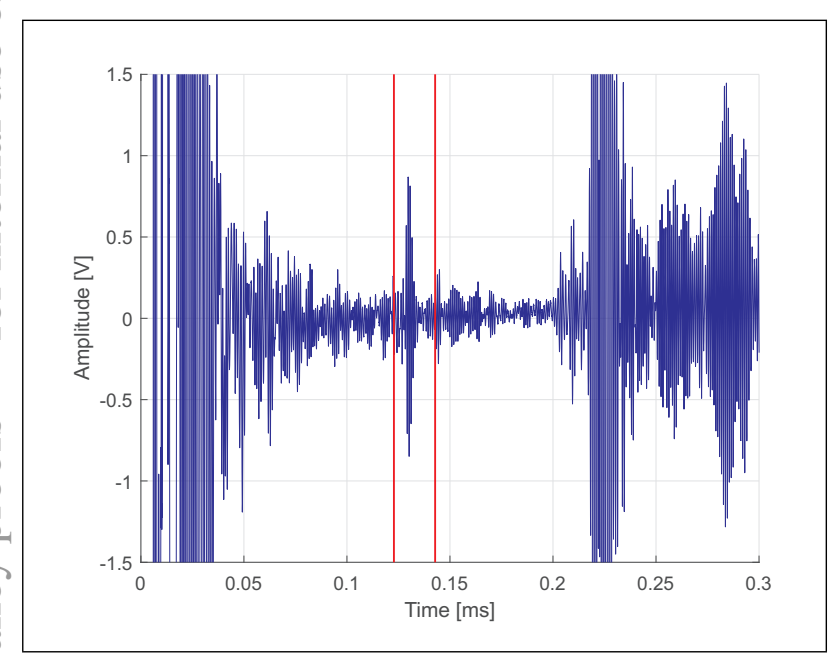

Figure 10. (Colour online) Typical time trace of the P/E measurement of the $10 \mathrm{~mm} \times 70 \mathrm{~mm}$ disbond, stand-off distance of $150 \mathrm{~mm}$ (wedge at $300 \mathrm{~mm}$ from plate end).

with an amplitude significantly above the noise level in the signal, but lower amplitude than the end reflection. The amplitude of this pulse was extracted for all pulse-echo measurements and is shown in the subsequent figures for the different defect cases. To quantify the damage detection sensitivity and uncertainty, measurements were conducted on two specimens manufactured without defects to quantify the baseline 'noise' level in the time traces, evaluating the amplitude in the time window corresponding to the arrival time of the expected reflected pulse.

Figure 11 shows the time trace for such a P/E measurement with the initial reverberations and end reflection clearly visible. Some baseline amplitude can be seen, which is not measurement noise, but coherent reflections either within the wedge or from manufacturing imperfections or other wave modes.

Figure 12a shows the baseline P/E amplitude results for two specimens without defects (two repetitions per specimen) as a function of the 'virtual' stand-off distance. 


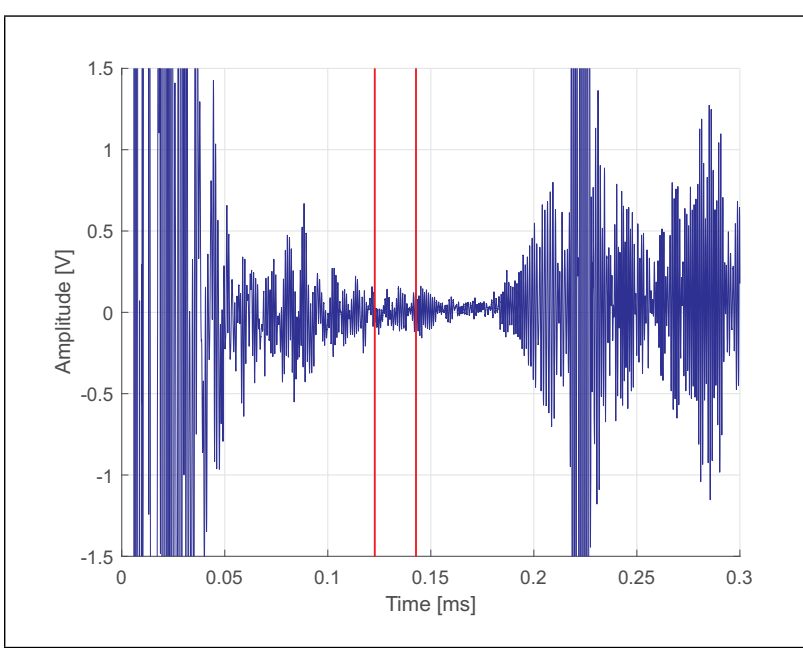

Figure 11. (Colour online) Typical time trace of the P/E measurement of a defect free plate, wedge at $300 \mathrm{~mm}$ from plate end.

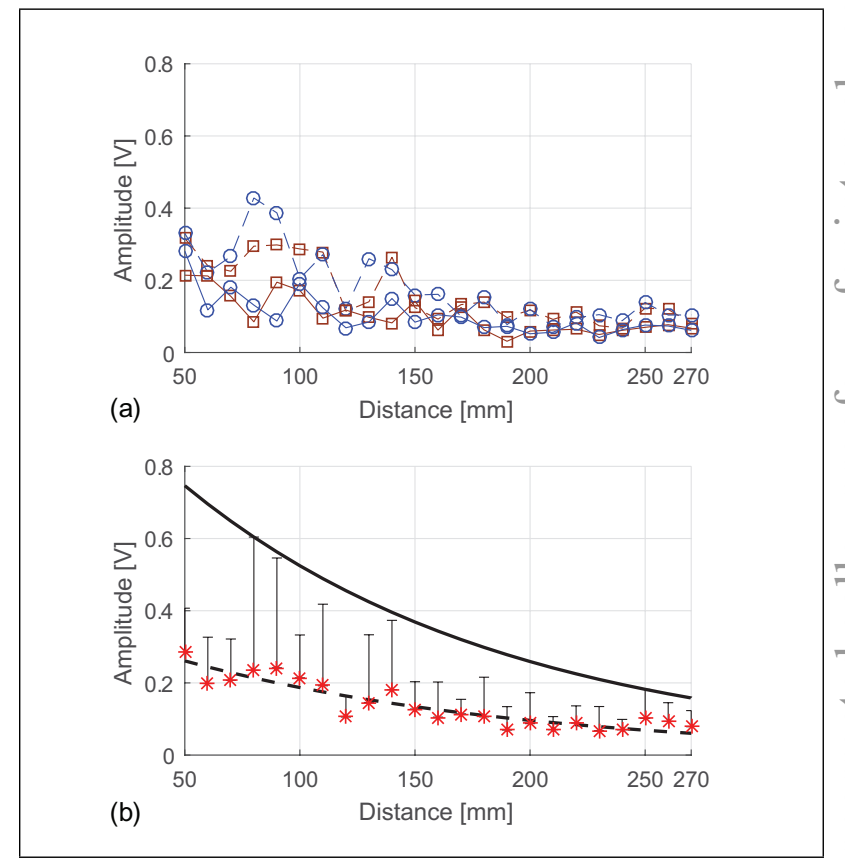

Figure 12. (Colour online) Measured P/E amplitudes for defect free specimens (baseline): a) individual measurements for two specimens (solid and dashed lines), 2 repetitions per specimen (squares and open points); b) average amplitude value for each stand-off distance (asterisks) with corresponding one-sided $95 \%$ confidence interval, exponential fit to average (dashed) and upper bound of $95 \%$ confidence interval (solid).

Except for high amplitudes for one measurement at 80 and $90 \mathrm{~mm}$, the baseline amplitudes follow approximately an exponential line with stand-off distance. The average pulse-echo amplitude and the one-sided 95\% confidence interval for each distance are displayed in Figure 12b. The confidence intervals were computed using Student's t-distribution to account for the small number of samples per distance (4 amplitude values). The best fit of an exponentially decreasing amplitude curve to the average value

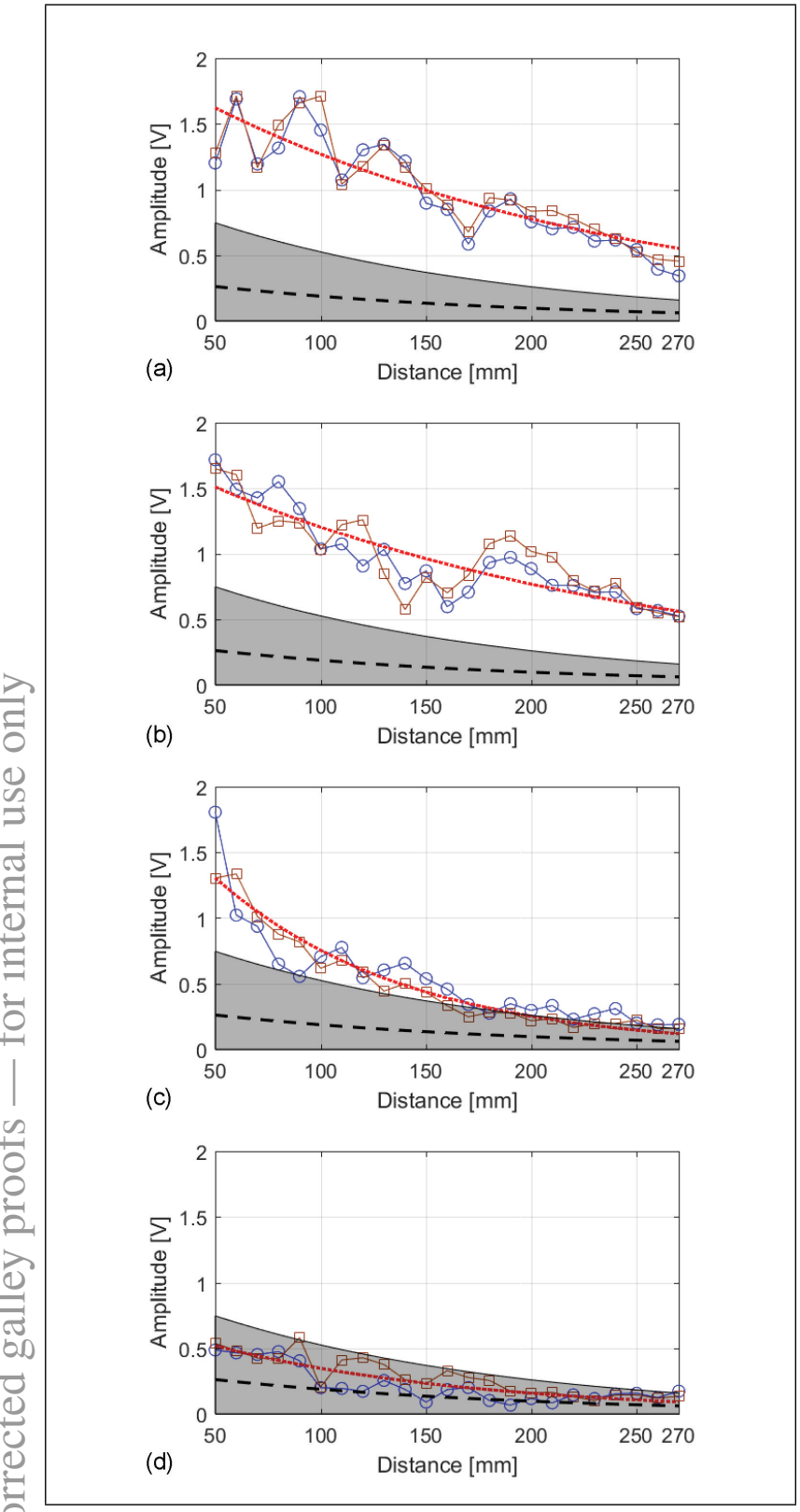

Figure 13. (Colour online) Amplitude of P/E measurements for 4 specimens with manufactured defects. 2 repetitions per defect (squares and open points) and exponential fit (dotted line). Exponential fit from baseline amplitude measurements (dashed line) and $95 \%$ confidence interval (grey area): a) $10 \mathrm{~mm} \times 70 \mathrm{~mm}$ lack of sealant; b) $10 \mathrm{~mm} \times 70 \mathrm{~mm}$ disbond; c) $15 \mathrm{~mm} \times 15 \mathrm{~mm}$ disbond; d) $10 \mathrm{~mm} \times 10 \mathrm{~mm}$ disbond.

and the upper bound of the $95 \%$ confidence interval are shown in Figure 12b.

Figure 13 shows the amplitude of the guided wave P/E measurements for the four different manufactured damages from different stand-off distances. While the amplitude for each measurement location varies slightly due to the manual placement of the wedge transducer with variations of the contact conditions and exact orientation, the amplitude levels are repeatable for each specimen. Measurements were repeated for each specimen and the best fit of an exponentially decreasing amplitude curve is shown. 
For the two larger damages across the specimen width (missing sealant, Figure 13a; disbond, Figure 13b) similar P/E amplitudes and dependency on the stand-off distance can be observed. Especially Figure 13a shows a periodic dependency of the amplitude on the stand-off distance, most likely due to interference effects of the different wave modes. This is also visible for the disbond (Figure $13 \mathrm{~b}$ ), but with slightly smaller variation compared to the fitted exponential curve.

The measurements for the larger square disbond (15 $\mathrm{mm} \times 15 \mathrm{~mm}$, Figure 13c) show an almost similar amplitude for short stand-off distances, but then a more significant drop in amplitude for longer propagation distance. As the width of the defect is limited, for longer stand-off distances only part of the incident wave is reflected at the defect. For the smaller manufactured square disbond $(10 \mathrm{~mm}$ $\times 10 \mathrm{~mm}$, Figure 13d) lower reflected amplitude was observed in line with the smaller defect size.

The $95 \%$ confidence interval and the exponential fit from the baseline measurements (Figure 12b) are overlaid on the measurement results in Figure 13.

For the two defects across the width of the specimen (missing sealant, disbond), the reflected P/E amplitude decreases by slightly more than half for an increase of the stand-off distance from $50 \mathrm{~mm}$ to $250 \mathrm{~mm}$ and is significantly above the baseline cases. Longer stand-off distances could not be realized in the experiments, but the observed behaviour indicates the potential for detection at longer distances. The larger square disbond ( $15 \mathrm{~mm} \times 15 \mathrm{~mm})$ could be detected for distances up to about $150 \mathrm{~mm}$ with $\mathrm{P} / \mathrm{E}$ amplitudes outside or at the border of the confidence interval. However, for distances over $150 \mathrm{~mm}$ the recorded amplitudes are comparable to the baseline case confidence line, and in a blind measurement it would be difficult to reliably detect this defect without more local measurements. The amplitude curves for the smaller square disbond $(10 \mathrm{~mm} \times 10 \mathrm{~mm})$ lie within the confidence interval of the baseline curve, indicating the limitations of the measurements. The methodology does not provide sufficient sensitivity to detect a defect this size from long stand- distances.

Figure 14 shows the results for the FE simulations for the cases of missing sealant and disbond. As the FE simulations were performed for a $2 \mathrm{D}$ geometry of the cross-section, this effectively compares to the experimental results for the defects across the specimen width, with spreading of the guided ultrasonic wave beam in the width direction being an additional consideration. The amplitude of the reflected $\mathrm{P} / \mathrm{E}$ pulse is shown on an arbitrary scale, as this cannot be directly related to the measurement voltage.

For both cases, smaller interference and local variations of the amplitude with stand-off distance are observed, and a difference in the amplitude for the two defect cases is predicted. This is in contrast to the measurement result, where the amplitudes were very similar. This is mainly due to the experimental conditions not accurately matching the assumed perfect line disbond as shown by the C-scan measurements. Compared to the measurement results a larger

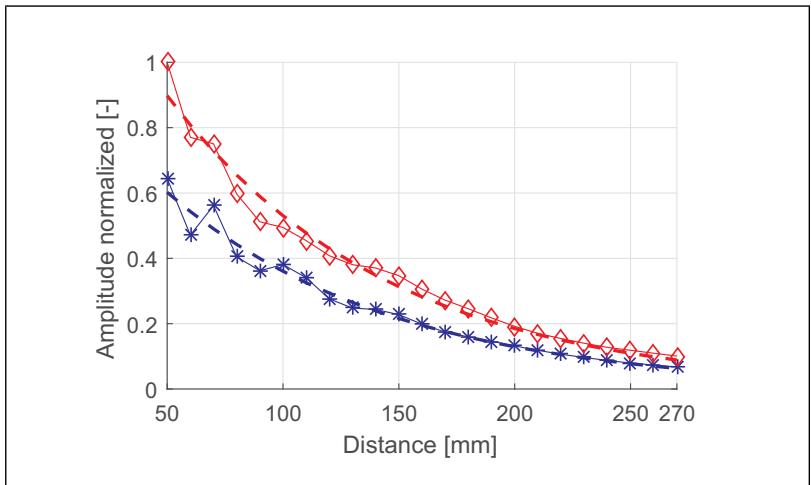

Figure 14. (Colour online) FE simulation results for predicted (arbitrary) amplitude of P/E pulse for missing sealant (stars) and disbond (diamonds) with exponential fits (dashed); 2D geometry similar to defects across specimen width.

drop in amplitude with stand-off distance is predicted. This results from the assumed amplitude drop due to damping and beam spread being larger than for the experimental case. The FE predictions therefore slightly underestimate the potential for defect detection for longer stand- off distances.

\section{Conclusions}

Defects within the bond layer of multi-layered metallic components were manufactured as missing sealant and localized disbonds. The uniformity of the bond layer and location of the hidden defects were verified from ultrasonic immersion C-scans. This standard non-destructive method allowed very good resolution of the defect shape and location, but required immersion of the specimen in the test rig. High frequency guided ultrasonic waves propagating along the specimen with insonification through the complete multi-layer specimen thickness were excited using standard wedge transducers. Interference occurs between the multiple Lamb wave modes during propagation along the structure, resulting in a frequency dependent variation of the energy through the thickness with distance. The reflection of the high frequency guided ultrasonic waves at small disbonds in the sealant layer and lack of sealant in the multilayer structure was measured using standard $\mathrm{P} / \mathrm{E}$ measurements. The detection sensitivity from a standoff distance was evaluated and compared to predictions from FE simulations. Defects across the specimen width could be clearly detected from long stand-off distances. For smaller disbonds and long stand-off distances, the amplitude values are comparable to the baseline case confidence line and in a blind measurement it would be difficult to reliably detect this defect without more local measurements.

\section{References}

[1] R. P. Dalton, P. Cawley, M. J. S. Lowe: The potential of guided waves for monitoring large areas of metallic aircraft fuselage structures. J. Nondestr. Eval. 20 (2001) 29-46.

[2] C. D. Smith: Nondestructive evaluation of aging aircraft, airports and aerospace hardware. - In: Proc. of SPIE 2945. R. D. Kempt, A. L. Broz (eds.). 1996, 200-209. 
[3] R. A. Smith, D. Edgard, L. D. Jones, D. M. Percivall: An ultrasonic solution for second- layer crack detection. Insight 47 (2005) 81-84.

[4] M. J. Santos, J. Perdigao, P. Faia: Ultrasonic guided waves scattering effects from defects in adhesively bonded lap joints using pitch and catch and pulse-echo techniques. J. Adhesion 84 (2008) 421-438.

[5] P. Fromme, P. D. Wilcox, M. J. S. Lowe, P. Cawley: On the development and testing of a guided ultrasonic wave array for structural integrity monitoring. IEEE Trans. Ultrason. Ferroelectr. Freq. Control 53 (2006) 777-785.

[6] D. N. Alleyne, P. Cawley: The interaction of Lamb waves with defects. IEEE Trans. Ultrason. Ferroelectr. Freq. Control 39 (1992) 381-397.

[7] M. Castaings, E. Le Clezio, B. Hosten: Modal decomposition method for modeling the interaction of Lamb waves with cracks. J. Acoust. Soc. Am. 112 (2002) 2567-2582.

[8] R. Seifried, L. J. Jacobs, J. Qu: Propagation of guided waves in adhesive bonded components. NDT\&E Int. 35 (2002) 317-328.

[9] Z. Fan, M. Castaings, M. J. S. Lowe, C. Biateau, P. Fromme: Feature-guided waves for monitoring adhesive shear modulus in bonded stiffeners. NDT\&E Int. 54 (2013) 96-102.

[10] N. Quaegebeur, P. Micheau, P. Masson, M. Castaings: Methodology for optimal configuration in structural health monitoring of composite bonded joints. Smart Mater. Struct. 21 (2012) 105001.

[11] H. Matt, I. Bartoli, F. L. di Scalea: Ultrasonic guided wave monitoring of composite wing skin-to-spar bonded joints in aerospace structures. J. Acoust. Soc. Am. 118 (2005) 2240-2252.

[12] E. Kostson, P. Fromme: Fatigue crack growth monitoring in multi-layered structures using guided ultrasonic waves. J. Phys.: Conf. Ser. 195 (2009) 012003.

[13] N. Terrien, D. Osmont, D. Royer, F. Lepoutre, A. Déom: A combined finite element and modal decomposition method to study the interaction of Lamb modes with micro-defects. Ultrasonics 46 (2007) 47-78.

[14] D. W. Greve, P. Zheng, I. J. Oppenheim: The transition from Lamb waves to longitudinal waves in plates. Smart Mater. Struct. 17 (2008) 035029.

[15] P. Khalili, P. Cawley: Excitation of single-mode Lamb waves at high-frequency-thickness products. IEEE Trans. Ultrason. Ferroelectr. Freq. Control 63 (2016) 303-312.

[16] D. Ratnam, K. Balasubramaniam, B. W. Maxfield: Generation and detection of higher-order mode clusters of guided waves (HOMC-GW) using meander-coil EMATs.
IEEE Trans. Ultrason. Ferroelectr. Freq. Control 59 (2012) 727-737.

[17] B. Masserey, P. Fromme: Surface defect detection in stiffened plate structures using Rayleigh-like wave. NDT\&E Int. 42 (2009) 564-572.

[18] E. Lindgren, J. C. Aldrin, K. Jata, B. Scholes, J. Knopp: Ultrasonic plate waves for fatigue crack detection in multilayered metallic structures. - In: Proc. of SPIE 6532, Health Monitoring of Structural and Biological Systems. T. Kundu (ed.). 2007, 653207 .

[19] P. B. Nagy, L. Adler: Nondestructive evaluation of adhesive joints by guided-waves. J. Appl. Phys. 66 (1989) 46584663.

[20] D. Cerniglia, A. Pantano, N. Montinaro: 3D simulations and experiments of guided wave propagation in adhesively bonded multi-layered structures. NDT\&E Int. 43 (2010) 527-535.

[21] H. Chan, B. Masserey, P. Fromme: High frequency guided ultrasonic waves for hidden fatigue crack growth monitoring in multi-layer model aerospace structures. Smart Mater. Struct. 24 (2015) 025037.

[22] A. Semoroz, B. Masserey, P. Fromme: Monitoring of hidden damage in multi-layered aerospace structures using high frequency guided waves. - In: Proc. of SPIE 7984, Health Monitoring of Structural and Biological Systems. T. Kundu (ed.). 2011, 79840T.

[23] B. Masserey, C. Raemy, P. Fromme: High-frequency guided ultrasonic waves for hidden defect detection in multilayered aircraft structures. Ultrasonics 54 (2014) 1720 1728.

[24] Henkel: Hysol EA 9394 epoxy paste adhesive property sheet. 2002.

[25] B. Pavlakovic, M. Lowe, D. Alleyne, P. Cawley: DISPERSE: A general purpose program for creating dispersion curves. - In: Rev. of Prog. in QNDE 16. D. O. Thompson, D. Chimenti (eds.). Plenum, New York, 1997, 185-192.

[26] D. Royer, E. Dieulesaint: Elastic waves in solids 1: Free and guided propagation. Springer, Berlin, 1999. p.317.

[27] B. Masserey, P. Fromme: On the reflection of coupled Rayleigh-like waves at surface defects in plates. J. Acoust. Soc. Am. 123 (2008) 88-98.

[28] J. Virieux: P-SV wave propagation in heterogeneous media: Velocity-stress finite-difference method. Geophysics 51 (1986) 889-901.

[29] B. Masserey, E. Mazza: Analysis of the near-field ultrasonic scattering at a surface crack. J. Acoust. Soc. Am. 118 (2005) 3585-3594. 